\title{
Modified Hamiltonian Formalism for Regge-Teitelboim Cosmology
}

\author{
Pinaki Patra, Md. Raju, Gargi Manna, and Jyoti Prasad Saha
}

Department of Physics, University of Kalyani, Kalyani 741235, India

Correspondence should be addressed to Pinaki Patra; monk.ju@gmail.com

Received 22 July 2014; Revised 17 November 2014; Accepted 8 December 2014; Published 28 December 2014

Academic Editor: Ashok Chatterjee

Copyright (C) 2014 Pinaki Patra et al. This is an open access article distributed under the Creative Commons Attribution License, which permits unrestricted use, distribution, and reproduction in any medium, provided the original work is properly cited.

\begin{abstract}
The Ostrogradski approach for the Hamiltonian formalism of higher derivative theory is not satisfactory because the Lagrangian cannot be viewed as a function on the tangent bundle to coordinate manifold. In this paper, we have used an alternative approach which leads directly to the Lagrangian which, being a function on the tangent manifold, gives correct equation of motion; no new coordinate variables need to be added. This approach can be used directly to the singular (in Ostrogradski sense) Lagrangian. We have used this method for the Regge-Teitelboim (RT) minisuperspace cosmological model. We have obtained the Hamiltonian of the dynamical equation of the scale factor of RT model.
\end{abstract}

\section{Introduction}

It is fairly well known that adding higher derivatives term in Lagrangian may improve the theory in some respects, like ultraviolet behavior [1,2], gravity renormalization [3], or even making modified gravity asymptotically free [4]. Also, higher-derivative Lagrangians appear to be a useful tool to describe some interesting models, like relativistic particles with rigidity, curvature, and torsion [5-10].

The starting of the study of higher derivative theory was started long back. Classical dynamics of a test particle's motion with higher-order time derivatives of the coordinates was first described in 1850 by Ostrogradski [11] and is known as Ostrogradski's formalism which has been extensively studied by several authors for its wide applicability [12-30].

An interesting occurrence of higher derivative terms in the action appears in general relativity. In some cases, such terms are isolated as surface terms and dropped. However, in the case of gravity, the surface term is never ignorable, for example, the requirement of the Gibbons-Hawking term in the action. This is more so in the brane world scenario where the universe is viewed as a hypersurface immersed in a bulk. A classic model is due to Regge and Teitelboim (RT) [31], where gravitation is described as the world volume swept out by the motion of a three-dimensional brane in a higher dimensional Minkowski spacetime. Hamiltonian analysis of the model and its quantization was further explored in [32-35]. Unlike the Einstein gravity, in the RT model, the independent fields are the embedding functions rather than the metric. In the RT model, second derivatives of the fields appear in the action, and like general relativity these higher derivative terms may be clubbed in a surface term. In the usual formulation, this surface term is dropped [35], thereby reducing the original model to a first-order theory. However, this makes the Hamiltonian formulation of the model problematic [35]. These problems are bypassed by introducing an auxiliary field [35]. On the other hand, recently it has been pointed out that no such auxiliary field is needed if one includes the surface term in the RT model containing higher derivative terms [34]. Obviously, therefore, the Hamiltonian formulation of this model is far from closed. The analysis of the RT model in the ambit of higher derivative theory [34] was done from the Ostrogradski approach and this work was based on the minisuperspace model following from the RT theory. The minisuperspace model carries the reparametrization invariance of the original RT gravity which appears as gauge invariance in the Hamiltonian analysis.

However, the main disadvantage of the Ostrogradski approach is that the Hamiltonian, being a linear function of some momenta, is necessarily unbounded from below. In 
general, this cannot be cured by trying to devise an alternative canonical formalism. In fact, any Hamiltonian is an integral of motion, while it is by far not obvious that a generic system described by higher derivative Lagrangians possesses globally defined integrals of motion, except the one related to time translation invariance. Moreover, the instability of the Ostrogradski Hamiltonian is not related to finite domains in phase space, which implies that it will survive in the standard quantization procedure (i.e., it cannot be cured by the uncertainty principle). The Ostrogradski approach also has some other disadvantages. There is no straightforward transition from the Lagrangian to the Hamiltonian formalism.

Recently, Andrzejewski et al. [36] have proposed a modified formalism for the higher derivative theory which can cure some of the drawbacks of Ostrogradski formalism. Basic idea is the same as that of Ostrogradski. But the advantage of this approach is that the Legendre transformation can be performed in a straightforward way, though the Hamiltonian of the modified formalism is directly connected to the Hamiltonian obtained in Ostrogradski formalism through a canonical transformation.

In this paper, we have used the modified formalism proposed by Andrzejewski et al. [36] for the Regge-Teitelboim (RT) minisuperspace cosmological model.

\section{Regge-Teitelboim Cosmological Model}

The Regge-Teitelboim cosmological model has been studied in [37]. We include this section for the completeness of our paper and we used their notation. The RT model considers a $d$-dimensional brane $\Sigma$ which evolves in an $N$-dimensional bulk spacetime with fixed Minkowski metric $\eta_{\mu \nu}$. The world volume swept out by the brane is a $d+1$-dimensional manifold $m$ defined by the embedding $x^{\mu}=X^{\mu} \zeta(a)$, where $x^{\mu}$ are the local coordinates of the background spacetime and $\zeta(a)$ are local coordinates for $m$. The theory is given by the action functional

$$
S[X]=\int_{m} d^{d+1} \zeta \sqrt{-g}\left(\frac{\beta}{2} \mathscr{R}-\Lambda\right),
$$

where $\beta$ has the dimension $[L]^{1-d}$ and $g$ is the determinant of the induced metric $g_{a b} . \Lambda$ denotes the cosmological constant and $R$ is the Ricci scalar. As has been already stated above, we will be confined to the minisuperspace cosmological model following from the RT model.

The standard procedure in cosmology is to assume that on the large scale the universe is homogeneous and isotropic. These special symmetries enable the four-dimensional world volume representing the evolving universe to be embedded in a five-dimensional Minkowski spacetime:

$$
d s^{2}=-d t^{2}+d a^{2}+a^{2} d \Omega_{3}^{2},
$$

where $d \Omega_{3}^{2}$ is the metric for unit 3 sphere. To ensure the FRW case, we take the following parametric representation for the brane:

$$
x^{\mu}=X^{\mu}\left(\zeta^{a}\right)=(t(\tau), a(\tau), \chi, \theta, \phi),
$$

where $a(\tau)$ is known as the scale factor.
After ADM decomposition [38, 39] with space-like unit normals $\left(N=\sqrt{\dot{t}^{2}-\dot{a}^{2}}\right.$ is the lapse function)

$$
n_{\mu}=\frac{1}{N}(-\dot{a}, \dot{t}, 0,0,0),
$$

the induced metric on the world volume is given by

$$
d s^{2}=-N^{2} d \tau^{2}+a^{2} d \Omega_{3}^{2} .
$$

Now, one can compute the Ricci scalar which is given by

$$
\mathscr{R}=\frac{6 \dot{t}}{a^{2} N^{4}}\left(a \ddot{a} \dot{t}-a \dot{a} \ddot{t}+N^{2} \dot{t}\right) .
$$

With these functions, we can easily construct the Lagrangian density as

$$
\mathscr{L}=\sqrt{-g}\left(\frac{\beta}{2} \mathscr{R}-\Lambda\right) .
$$

The Lagrangian in terms of arbitrary parameter $\tau$ can be written as [34]

$$
\mathscr{L}(a, \dot{a}, \ddot{a}, \dot{t}, \ddot{t})=\frac{a \dot{t}}{N^{3}}\left(a \ddot{a} \dot{t}-a \ddot{a} \ddot{t}+N^{2} \dot{t}\right)-N a^{3} H^{2},
$$

where

$$
H^{2}=\frac{\Lambda}{3 \beta} \text {. }
$$

$H$ is called the Hubble parameter.

Varying the action with respect to $a(\tau)$, we get the corresponding Euler-Lagrange equation:

$$
\frac{d}{d \tau}\left(\frac{\dot{a}}{\dot{t}}\right)=-\frac{N^{2}\left(\dot{t}^{2}-3 N^{2} a^{2} H^{2}\right)}{a \dot{t}\left(3 \dot{t}^{2}-N^{2} a^{2} H^{2}\right)} .
$$

Please note that the Lagrangian contains higher derivative terms of field $a$. However, we can write it as [34]

$$
L=-\frac{a \dot{a}^{2}}{N}+a N\left(1-a^{2} H^{2}\right)+\frac{d}{d \tau}\left(\frac{a^{2} \dot{a}}{N}\right) .
$$

If we neglect the boundary term, the resulting Lagrangian becomes the usual first-order one. However, the Hamiltonian analysis is facilitated if we retain the higher derivative term. Thus our Hamiltonian analysis will proceed from the above equation containing higher derivative term. Note that the higher-order model was also considered in [34], where the Hamiltonian analysis was performed following the Ostrogradski approach. We, on the contrary, will follow the equivalent first-order approach of Andrzejewski et al. [36].

\section{Hamiltonian Formalism for Regge-Teitelboim Cosmological Model}

Our concerned Lagrangian as mentioned in the previous section is

$$
L(a, \dot{a}, \ddot{a}, \dot{t}, \ddot{t})=\frac{a \dot{t}}{N^{3}}\left(a \ddot{a} \dot{t}-a \dot{a} \ddot{t}+N^{2} \dot{t}\right)-N a^{3} H^{2},
$$


where

$$
N=\sqrt{\dot{t}-\dot{a}^{2}}
$$

and the Hubble parameter which we are considering to be a constant in the present discussion is

$$
H^{2}=\frac{\Lambda}{3 \beta}
$$

We set, for the notational convenience,

$$
\begin{array}{lll}
a=q_{1}^{1}, & t=q_{1}^{2}, & \dot{a}=\dot{q}_{1}^{1}, \\
\dot{t}=\dot{q}_{1}^{2}, & \ddot{a}=q_{2}^{1}, & \ddot{t}=q_{2}^{2} .
\end{array}
$$

Our Lagrangian is singular in Ostrogradski sense because

$$
\begin{aligned}
\operatorname{det}\left(W_{\mu \nu}\right)=\operatorname{det}\left(\frac{\partial^{2} L}{\partial \ddot{q}^{\mu} \partial \ddot{q}^{\nu}}\right) & =\operatorname{det}\left[\begin{array}{cc}
\frac{\partial^{2} L}{\partial \ddot{a} \partial \ddot{a}} & \frac{\partial^{2} L}{\partial \ddot{\partial} \partial \ddot{t}} \\
\frac{\partial^{2} L}{\partial \ddot{t} \partial \ddot{a}} & \frac{\partial^{2} L}{\partial \ddot{\partial} \partial \ddot{t}}
\end{array}\right] \\
& =\operatorname{det}\left[\begin{array}{ll}
0 & 0 \\
0 & 0
\end{array}\right]=0 .
\end{aligned}
$$

Now, we define $F\left(q_{1}^{1}, q_{1}^{2}, \dot{q}_{1}^{1}, \dot{q}_{1}^{2}, q_{3}^{1}, q_{3}^{2}\right)$ such that

$$
\operatorname{det}\left(\frac{\partial^{2} F}{\partial \dot{q}_{1}^{\mu} \partial q_{3}^{\nu}}\right)=\left[\begin{array}{cc}
\frac{\partial^{2} F}{\partial \dot{q}_{1}^{1} \partial q_{3}^{1}} & \frac{\partial^{2} F}{\partial \dot{q}_{1}^{1} \partial q_{3}^{2}} \\
\frac{\partial^{2} F}{\partial \dot{q}_{1}^{2} \partial q_{3}^{1}} & \frac{\partial^{2} F}{\partial \dot{q}_{1}^{2} \partial q_{3}^{2}}
\end{array}\right] \neq 0 .
$$

One possible choice is

$$
\begin{aligned}
F\left(q_{1}^{1}, q_{1}^{2}, \dot{q}_{1}^{1}, \dot{q}_{1}^{2}, q_{3}^{1}, q_{3}^{2}\right) & =\alpha\left(\dot{q}_{1}^{1} q_{3}^{1}+\dot{q}_{1}^{2} q_{3}^{2}\right)+\beta\left(\dot{q}_{1}^{1} q_{3}^{2}+\dot{q}_{1}^{2} q_{3}^{1}\right) ; \\
\Delta & =\alpha^{2}-\beta^{2} \neq 0 .
\end{aligned}
$$

Now, using the suggestions prescribed in [36], we define

$$
\begin{aligned}
\mathscr{L}( & \left(q_{1}^{1}, q_{1}^{2}, \dot{q}_{1}^{1}, \dot{q}_{1}^{2}, q_{2}^{1}, q_{2}^{2}, q_{3}^{1}, q_{3}^{2}, \dot{q}_{3}^{1}, \dot{q}_{3}^{2}\right) \\
= & L+\frac{\partial F}{\partial q_{1}^{\mu}} \dot{q}_{1}^{\mu}+\frac{\partial F}{\partial q_{3}^{\mu}} \dot{q}_{3}^{\mu}+\frac{\partial F}{\partial \dot{q}_{1}^{\mu}} q_{2}^{\mu} \\
= & \frac{q_{1}^{1} \dot{q}_{1}^{2}}{N^{3}}\left(q_{1}^{1} q_{2}^{1} \dot{q}_{1}^{2}-q_{1}^{1} \dot{q}_{1}^{1} q_{2}^{2}+N^{2} \dot{q}_{1}^{2}\right)-N\left(q_{1}^{1}\right)^{3} H^{2} \\
& +\left(\alpha \dot{q}_{1}^{1}+\beta \dot{q}_{1}^{2}\right) \dot{q}_{3}^{1}+\left(\beta \dot{q}_{1}^{1}+\alpha \dot{q}_{1}^{2}\right) \dot{q}_{3}^{2}+q_{2}^{1}\left(\alpha q_{3}^{1}+\beta q_{3}^{2}\right) \\
& +\left(\alpha q_{3}^{2}+\beta q_{3}^{1}\right) q_{2}^{2} .
\end{aligned}
$$

The conjugate momenta corresponding to the coordinates are given by $p_{i j}=\partial \mathscr{L} / \partial \dot{q}_{i}^{j}$. In particular,

$$
\begin{gathered}
p_{11}=\frac{3}{N^{5}}\left(q_{1}^{1}\right)^{2} \dot{q}_{1}^{1} \dot{q}_{1}^{2}\left(q_{2}^{1} \dot{q}_{1}^{2}-\dot{q}_{1}^{1} q_{2}^{2}\right)+\frac{q_{1}^{1} \dot{q}_{1}^{2}}{N^{3}}\left(\dot{q}_{1}^{1} \dot{q}_{1}^{2}-q_{1}^{1} q_{2}^{2}\right) \\
-\frac{H^{2}}{N} \dot{q}_{1}^{1}\left(q_{1}^{1}\right)^{3}+\alpha \dot{q}_{3}^{1}+\beta \dot{q}_{3}^{2} \\
p_{12}=\frac{3\left(q_{1}^{1} \dot{q}_{1}^{2}\right)^{2}}{N^{5}}\left(\dot{q}_{1}^{1} q_{2}^{2}-q_{2}^{1} \dot{q}_{1}^{2}\right) \\
+\frac{q_{1}^{1}}{N^{3}}\left(2 q_{1}^{1} q_{2}^{1} \dot{q}_{1}^{2}-q_{1}^{1} \dot{q}_{1}^{1} q_{2}^{2}-\left(\dot{q}_{1}^{2}\right)^{3}\right) \\
+\frac{q_{1}^{1}}{N}\left(2 \dot{q}_{1}^{2}-H^{2}\left(q_{1}^{1}\right)^{2} \dot{q}_{1}^{2}\right)+\left(\alpha \dot{q}_{3}^{2}+\beta \dot{q}_{3}^{1}\right) \\
p_{21}=0 \\
p_{22}=0 \\
p_{31}=\frac{\partial F}{\partial q_{3}^{1}}=\alpha \dot{q}_{1}^{1}+\beta \dot{q}_{1}^{2} \\
p_{32}=\frac{\partial F}{\partial q_{3}^{2}}=\beta \dot{q}_{1}^{1}+\alpha \dot{q}_{1}^{2}
\end{gathered}
$$

Equations (22) and (23) provide primary constraint. Equation (17) enables us to solve $\dot{q}$ 's in terms of momenta. These are explicitly given by

$$
\begin{array}{cl}
\dot{q}_{1}^{1}=\frac{1}{\Delta} P_{31}, & \dot{q}_{1}^{2}=\frac{1}{\Delta} P_{32}, \\
\dot{q}_{3}^{1}=\frac{\alpha \varrho_{1}-\beta \varrho_{2}}{\Delta}, & \dot{q}_{3}^{2}=\frac{\alpha \varrho_{2}-\beta \varrho_{1}}{\Delta},
\end{array}
$$

where

$$
\begin{gathered}
P_{31}=\alpha p_{31}-\beta p_{32}, \\
P_{32}=\left(\alpha p_{32}-\beta p_{31}\right), \\
\varrho_{1}=p_{11}+\frac{1}{N \Delta} H^{2}\left(q_{1}^{1}\right)^{3} P_{31}-\frac{q_{1}^{1}}{N^{3} \Delta} P_{32}\left[\frac{1}{\Delta^{2}} P_{31} P_{32}-q_{1}^{1} q_{2}^{2}\right] \\
-\frac{3\left(q_{1}^{1}\right)^{2}}{N^{5} \Delta^{3}} P_{31} P_{32}\left[q_{2}^{1} P_{32}-q_{2}^{2} P_{31}\right], \\
\varrho_{2}=p_{12}+\frac{\left(q_{1}^{1} P_{32}\right)^{2}}{\Delta}\left(q_{2}^{1} P_{32}-q_{2}^{2} P_{31}\right)+\frac{q_{1}^{1}}{N^{3} \Delta} \\
\times\left[q_{1}^{1} P_{31} q_{2}^{2}+\frac{1}{\Delta^{2}} P_{32}^{3}-2 q_{1}^{1} q_{2}^{1} P_{32}\right] \frac{q_{1}^{1}}{N \Delta} \\
\times\left[H^{2}\left(q_{1}^{1}\right)^{2}-2\right] P_{32} .
\end{gathered}
$$


Therefore, $N$ reduces to

$$
N=\sqrt{\frac{\left(p_{32}\right)^{2}-\left(p_{31}\right)^{2}}{\Delta}} .
$$

Now, the Dirac Hamiltonian $(\mathscr{H})$ is given by

$$
\mathscr{H}=p_{1 \mu} \dot{q}_{1}^{\mu}-L-\frac{\partial F}{\partial q_{1}^{\mu}} \dot{q}_{1}^{\mu}-\frac{\partial F}{\partial \dot{q}_{1}^{\mu}} q_{2}^{\mu}+c^{\mu} p_{2 \mu},
$$

where $c^{\mu}$ are two Lagrange multipliers enforcing the "primary" constraints

$$
\Phi_{1 \mu} \equiv p_{2 \mu} \approx 0
$$

The Hamiltonian explicitly reads

$$
\begin{aligned}
\mathscr{H}= & \frac{1}{\Delta}\left(p_{11} P_{31}+p_{12} P_{32}\right)-\frac{1}{N \Delta^{2}} q_{1}^{1}\left(P_{32}\right)^{2} \\
& -\frac{1}{N^{3} \Delta^{2}}\left[\left(q_{1}^{1}\right)^{2} q_{2}^{1}\left(P_{32}\right)^{2}-\left(q_{1}^{1}\right)^{2} q_{2}^{2} P_{31} P_{32}\right] \\
& -\alpha\left(q_{3}^{1} q_{2}^{1}+q_{3}^{2} q_{2}^{2}\right)-\beta\left(q_{3}^{2} q_{2}^{1}+q_{3}^{1} q_{2}^{2}\right)+H^{2} N\left(q_{1}^{1}\right)^{3} \\
& +c^{1} p_{21}+c^{2} p_{22} .
\end{aligned}
$$

And the Hamilton equations of motion are given by

$$
\begin{gathered}
\dot{q}_{1}^{1}=\frac{1}{\Delta} P_{31}, \\
\dot{p}_{11}=\frac{1}{N \Delta^{2}}\left(P_{32}\right)^{2}+\frac{2 q_{1}^{1}}{N^{3} \Delta^{2}}\left(q_{2}^{1} P_{32}-q_{2}^{2} P_{31}\right) P_{32} \\
-3 H^{2} N\left(q_{1}^{1}\right)^{2}, \\
\dot{q}_{1}^{2}=\frac{1}{\Delta} P_{32}, \quad \dot{p}_{12}=0, \quad \dot{q}_{2}^{1}=c^{1}, \\
\dot{p}_{21}=\frac{1}{N^{3} \Delta^{2}}\left(q_{1}^{1} P_{32}\right)^{2}+\alpha q_{3}^{1}+\beta q_{3}^{2}, \\
\dot{q}_{3}^{1}=\frac{\dot{q}_{2}^{2}=c^{2},}{\Delta}=\frac{1}{N^{3} \Delta^{2}}\left(q_{1}^{1}\right)^{2} P_{31} P_{32}+\alpha q_{3}^{2}+\beta q_{3}^{1}, \\
+\frac{2 \beta}{N \Delta^{2}} q_{1}^{1} P_{32}-\frac{3 p_{31}}{N^{5} \Delta^{3}}\left(q_{1}^{1}\right)^{2} P_{32}\left[q_{2}^{1} P_{32}-q_{2}^{2} P_{31}\right] \\
-\frac{1}{N^{3} \Delta^{2}}\left(q_{1}^{1}\right)^{2}\left[\beta q_{2}^{2} P_{31}-\left(\alpha q_{2}^{2}+2 \beta q_{2}^{1}\right) P_{32}\right] \\
-\frac{1}{N \Delta} H^{2} p_{31}\left(q_{1}^{1}\right)^{3}, \\
\left.p_{31}\right)^{2}
\end{gathered}
$$

$$
\begin{gathered}
\dot{p}_{31}=\alpha q_{2}^{1}+\beta q_{2}^{2} \\
\dot{q}_{3}^{2}=\frac{1}{\Delta}\left(\alpha p_{12}-\beta p_{11}\right)+\frac{1}{N^{3} \Delta^{3}} q_{1}^{1} p_{32}\left(P_{32}\right)^{2} \\
-\frac{2 \alpha}{N \Delta^{2}} q_{1}^{1} P_{32}+\frac{3 p_{32}}{N^{5} \Delta^{3}}\left(q_{1}^{1}\right)^{2} P_{32}\left[q_{2}^{1} P_{32}-q_{2}^{2} P_{31}\right] \\
-\frac{1}{N^{3} \Delta^{2}}\left(q_{1}^{1}\right)^{2}\left[-\alpha q_{2}^{2} P_{31}+\left(2 \alpha q_{2}^{1}+\beta q_{2}^{2}\right) P_{32}\right] \\
+\frac{1}{N \Delta} H^{2} p_{32}\left(q_{1}^{1}\right)^{3}, \\
\dot{p}_{32}=\alpha q_{2}^{2}+\beta q_{2}^{1} .
\end{gathered}
$$

The secondary constraints read

$$
0 \approx \Phi_{2 \mu}=\frac{\partial L\left(q_{1}, \dot{q}_{1}, q_{2}\right)}{\partial q_{2}^{\mu}}+\frac{\partial F\left(q_{1}, \dot{q}_{1}, q_{3}\right)}{\partial \dot{q}_{1}^{\mu}}
$$

that is,

$$
\begin{gathered}
0 \approx \Phi_{21}=\frac{1}{N^{3}}\left(q_{1}^{1} \dot{q}_{1}^{2}\right)^{2}+\alpha q_{3}^{1}+\beta q_{3}^{2}, \\
0 \approx \Phi_{22}=-\frac{1}{N^{3}}\left(q_{1}^{1}\right)^{2} \dot{q}_{1}^{1} \dot{q}_{1}^{2}+\alpha q_{3}^{2}+\beta q_{3}^{1} .
\end{gathered}
$$

To determine $c^{\mu}$, usually one uses the stability condition of the secondary constraints:

$$
0 \approx\left\{\Phi_{2 \mu}, \mathscr{H}\right\}
$$

But, for our system under consideration, $W$ in (16) has rank 0 . This implies the existence of 2 linearly independent null vectors; so one cannot obtain the values of $c^{\mu}$ in this case. However, if we are interested in the dynamical equations for $a$ and $t$, we can use the Hamiltonian

$$
\mathscr{H}=p_{1 \mu} \dot{q}_{1}^{\mu}-L-\frac{\partial F}{\partial q_{1}^{\mu}} \dot{q}_{1}^{\mu}-\frac{\partial F}{\partial \dot{q}_{1}^{\mu}} q_{2}^{\mu}
$$

which is explicitly given by

$$
\begin{aligned}
\mathscr{H}= & \frac{1}{\Delta}\left(p_{11} P_{31}+p_{12} P_{32}\right)-\frac{1}{N \Delta^{2}} q_{1}^{1}\left(P_{32}\right)^{2}-\frac{1}{N^{3} \Delta^{2}} \\
& \times\left[\left(q_{1}^{1}\right)^{2} q_{2}^{1}\left(P_{32}\right)^{2}-\left(q_{1}^{1}\right)^{2} q_{2}^{2} P_{31} P_{32}\right] \\
& -\alpha\left(q_{3}^{1} q_{2}^{1}+q_{3}^{2} q_{2}^{2}\right)-\beta\left(q_{3}^{2} q_{2}^{1}+q_{3}^{1} q_{2}^{2}\right)+H^{2} N\left(q_{1}^{1}\right)^{3} .
\end{aligned}
$$

If we promote it to quantization, it can easily be seen that $\mathscr{H}$ is hermitian.

\section{Conclusions}

We have obtained the Hamiltonian structure for the scale factor of the RT model. In the modified formalism used in this paper, the Legendre transformation can be performed in 
a straightforward way. Summarizing, we have found modified Hamiltonian formulations of RT gravity which is equivalent to the Ostrogradski formalism in the sense that they are related to the latter by a canonical transformation.

The stability condition of constraint for the modified formalism proposed in [36] fails to determine the lagrange multipliers for the model discussed in this paper. In that sense, one can conclude that the modified formalism proposed in [36] is not always superior to the usual Ostrogradski formalism used in literature.

\section{Conflict of Interests}

The authors declare that there is no conflict of interests regarding the publication of this paper.

\section{Acknowledgments}

The authors would like to thank the anonymous reviewers for their careful reading of the paper and for their valuable comments which made this paper in the present form. Pinaki Patra is grateful for CSIR, Government of India, for fellowship support; Gargi Manna is grateful for DST, Government of India, for DST-INSPIRE scholarship; and Jyoti Prasad Saha is grateful for DST-PURSE for financial support.

\section{References}

[1] W. Thiring, "Regularization as a consequence of higher order equations," Physical Review, vol. 77, p. 570, 1950.

[2] A. Pais and G. E. Uhlenbeck, "On field theories with non-localized action," Physical Review Letters, vol. 79, pp. 145-165, 1950.

[3] K. S. Stelle, "Renormalization of higher-derivative quantum gravity," Physical Review D, vol. 16, no. 4, pp. 953-969, 1977.

[4] E. S. Fradkin and A. A. Tseytlin, "Renormalizable asymptotically free quantum theory of gravity," Nuclear Physics. B, vol. 201, no. 3, pp. 469-481, 1982.

[5] Y. A. Kuznetsov and M. S. Plyushchay, "The model of the relativistic particle with curvature and torsion," Nuclear Physics B, vol. 389, no. 1, pp. 181-205, 1993.

[6] R. D. Pisarski, "Field theory of paths with a curvature-dependent term," Physical Review. D. Particles and Fields. Third Series, vol. 34, no. 2, pp. 670-673, 1986.

[7] V. V. Nesterenko, "Singular Lagrangians with higher derivatives," Journal of Physics. A. Mathematical and General, vol. 22, no. 10, pp. 1673-1687, 1989.

[8] M. S. Plyushchay, "Massive relativistic point particle with rigidity," International Journal of Modern Physics A, vol. 4, p. 3851, 1989.

[9] M. S. Plyushchay, "The model of the relativistic particle with torsion," Nuclear Physics B, vol. 362, no. 1-2, pp. 54-72, 1991.

[10] R. Banerjee, P. Mukherjee, and B. Paul, "Gauge symmetry and W-algebra in higher derivative systems," Journal of High Energy Physics, vol. 2011, article 85, 2011.

[11] M. Ostrogradski, Mémoires de l'Académie Impériale des Sciences de Saint-Pétersbourg, Series 4, 1850.

[12] B. Podolsky, "A generalized electrodynamics. I. Nonquantum," Physical Review, vol. 62, pp. 68-71, 1942.
[13] B. Podolsky and C. Kikuchi, "A generalized electrodynamics part II-quantum," Physical Review, vol. 65, p. 228, 1944.

[14] B. Podolsky and C. Kikuchi, "Auxiliary conditions and electrostatic interaction in generalized quantum electrodynamics," vol. 67, pp. 184-192, 1945.

[15] J. Iliopoulos and B. Zumino, "Broken supergauge symmetry and renormalization," Nuclear Physics B, vol. 76, no. 2, pp. 310-332, 1974.

[16] D. A. Eliezer and R. P. Woodard, "The problem of nonlocality in string theory," Nuclear Physics B, vol. 325, no. 2, pp. 389-469, 1989.

[17] I. P. Neupane, "Consistency of higher derivative gravity in the brane background," Journal of High Energy Physics, vol. 2000, no. 9, article 040, 2000.

[18] S. Nojiri, S. D. Odintsov, and S. Ogushi, "Cosmological and black hole brane-world universes in higher derivative gravity," Physical Review D, vol. 65, Article ID 023521, 2001.

[19] C.-S. Chu, J. Lukierski, and W. J. Zakrzewski, "Hermitian analyticity, IR/UV mixing and unitarity of noncommutative field theories," Nuclear Physics. B, vol. 632, no. 1-3, pp. 219-239, 2002.

[20] S. M. Carroll, M. Hoffman, and M. Trodden, "Can the dark energy equation-of-state parameter $w$ be less than -1?" Physical Review D, vol. 68, Article ID 023509, 2003.

[21] A. Anisimov, E. Babichev, and A. Vikman, "B-inflation," Journal of Cosmology and Astroparticle Physics, vol. 2005, no. 06, 2005.

[22] R. P. Woodard, "Avoiding dark energy with $1 / \mathrm{R}$ modifications of gravity," in The Invisible Universe: Dark Matter and Dark Energy, vol. 720 of Lecture Notes in Physics, pp. 403-433, Springer, Berlin, Germany, 2007.

[23] R. Cordero, A. Molgado, and E. Rojas, "Ostrogradski approach for the Regge-Teitelboim type cosmology," Physical Review DParticles, Fields, Gravitation, and Cosmology, vol. 79, no. 2, Article ID 024024, 10 pages, 2009.

[24] R. Andringa, E. A. Bergshoeff, M. De Roo, O. Hohm, E. Sezgin, and P. K. Townsend, "Massive 3D supergravity," Classical and Quantum Gravity, vol. 27, no. 2, Article ID 025010, 2010.

[25] E. A. Bergshoeff, O. Hohm, J. Rosseel, E. Sezgin, and P. K. Townsend, "More on massive 3D supergravity," Classical and Quantum Gravity, vol. 28, no. 1, Article ID 015002, 2011.

[26] F. S. Gama, M. Gomes, J. R. Nascimento, A. Yu. Petrov, and A. J. da Silva, "Higher-derivative supersymmetric gauge theory," Physical Review D, vol. 84, Article ID 045001, 2011.

[27] P. Mukherjee and B. Paul, "Gauge invariances of higher derivative Maxwell-Chern-Simons field theory-a new Hamiltonian approach," Physical Review D, vol. 85, no. 4, Article ID 045028, 2012.

[28] A. Escalante, J. Guven, E. Rojas, and R. Capovilla, "Hamiltonian dynamics of extended objects: Regge-Teitelboim model," International Journal of Theoretical Physics, vol. 48, pp. 2486-2498, 2009.

[29] R. Cordero, M. Cruz, A. Molgado, and E. Rojas, "Quantum modified Regge-Teitelboim cosmology," General Relativity and Gravitation, vol. 46, no. 7, p. 1761, 2014, Erratum in General Relativity and Gravitation, vol. 46, no. 8, p. 1770, 2014.

[30] R. Cordero, A. Molgado, and E. Rojas, "Quantum charged rigid membrane," Classical and Quantum Gravity, vol. 28, no. 6, Article ID 065010, 2011.

[31] T. Regge and C. Teitelboim, in Proceedings of the Marcel Grossman Meeting, R. Ruffini, Ed., North-Holland, Trieste, Italy, 1977.

[32] A. Davidson, D. Karasik, and Y. Lederer, "Wavefunction of a brane-like universe," Classical Quantum Gravity, vol. 16, no. 4, pp. 1349-1356, 1999. 
[33] A. Davidson, D. Karasik, and Y. Lederer, "Geodesic evolution and nucleation of a de Sitter brane," Physical Review D, vol. 72, no. 6, Article ID 064011, 5 pages, 2005.

[34] R. Cordero, A. Molgado, and E. Rojas, "Ostrogradski approach for the Regge-Teitelboim type cosmology," Physical Review D, vol. 79, no. 2, Article ID 024024, 2009.

[35] D. Karasik and A. Davidson, "Geodetic brane gravity," Physical Review D, vol. 67, no. 6, 2003.

[36] K. Andrzejewski, J. Gonera, P. MacHalski, and P. Maślanka, "Modified Hamiltonian formalism for higher-derivative theories," Physical Review D-Particles, Fields, Gravitation and Cosmology, vol. 82, no. 4, Article ID 045008, 2010.

[37] R. Banerjee, P. Mukherjee, and B. Paul, "New Hamiltonian analysis of Regge-Teitelboim minisuperspace cosmology," Physical Review D-Particles, Fields, Gravitation and Cosmology, vol. 89, no. 4, Article ID 043508, 2014.

[38] R. Arnowitt, S. Deser, and C. W. Misner, "Republication of: the dynamics of general relativity," General Relativity and Gravitation, vol. 40, no. 9, pp. 1997-2027, 2008.

[39] S. Deser, F. A. E. Pirani, and D. C. Robinson, "New embedding model of general relativity," Physical Review D, vol. 14, no. 12, pp. 3301-3303, 1976. 

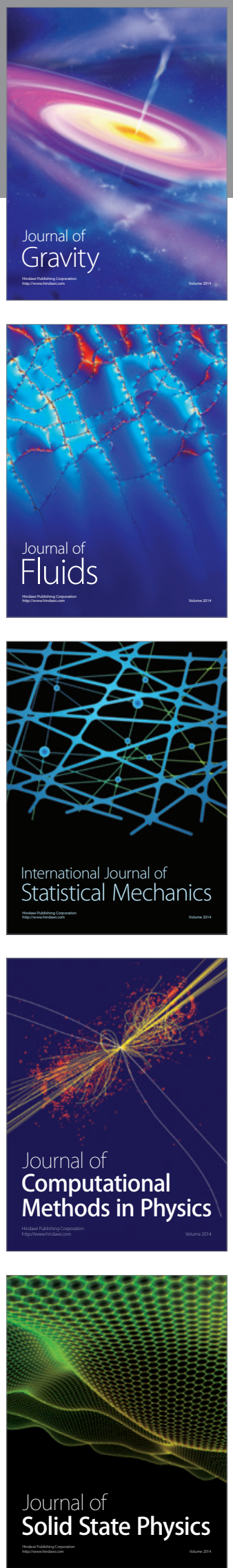

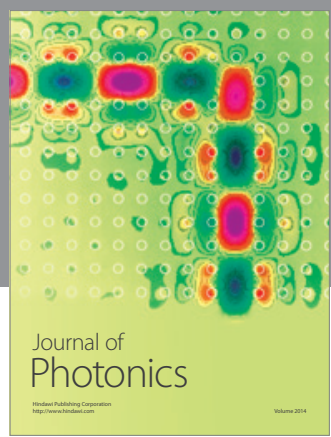

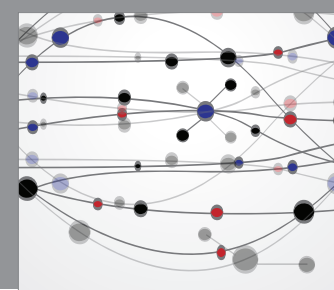

The Scientific World Journal

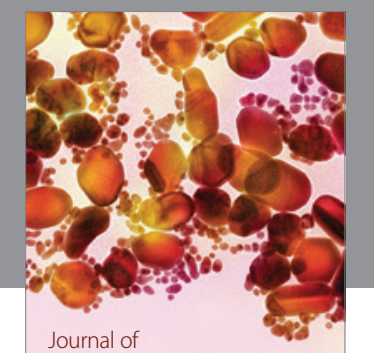

Soft Matter
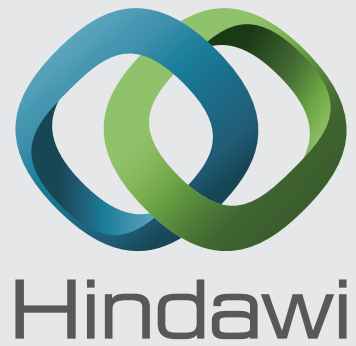

Submit your manuscripts at

http://www.hindawi.com
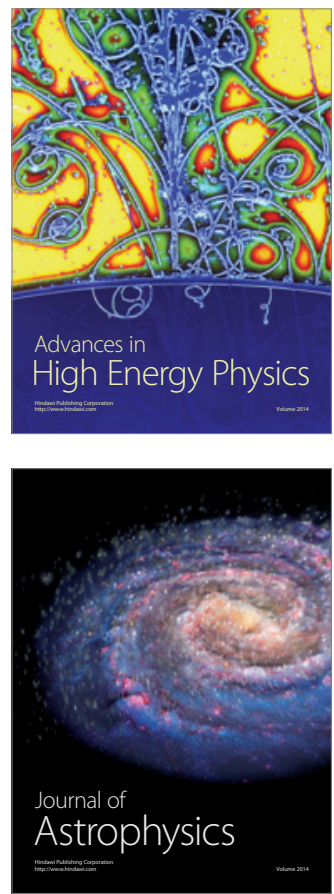
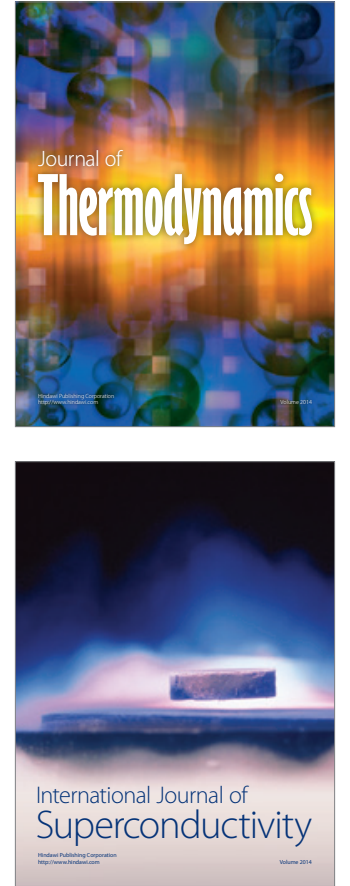
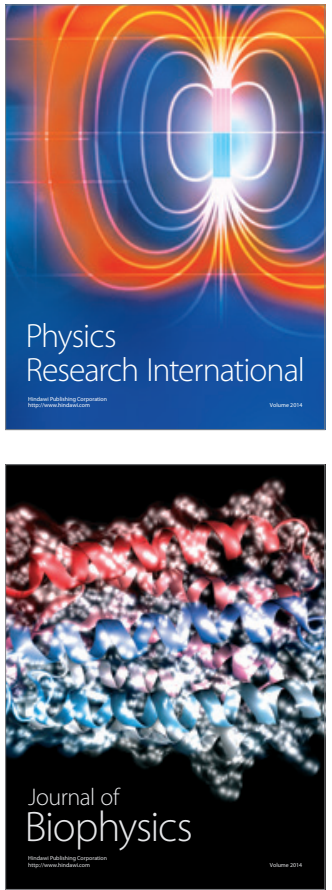
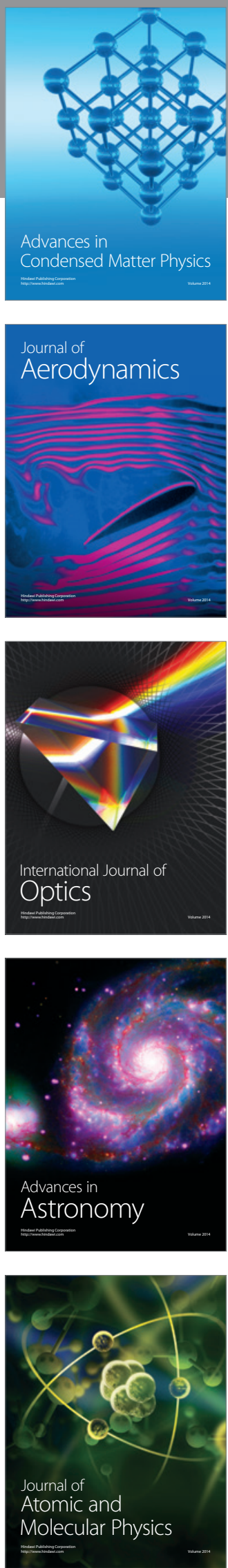